\title{
La paulatina adopción de ORCID para la mejora de la identidad digital de las revistas científicas españolas en acceso abierto
}

\author{
Francisco-Javier Martínez-Méndez* \\ Rosana López-Carreño*
}

\begin{abstract}
Artículo recibido:
24 de julio de 2018

Artículo aceptado:

10. de enero de 2019
\end{abstract}

Artículo de revisión

\section{Resumen}

Este trabajo reflexiona sobre la necesidad de adoptar un identificador digital para autores o investigadores, el cual facilite el control de autoridades, así como para la actualización de la currícula y la determinación de métricas en la producción científica, concluyendo que el ORCID puede ser el indicador más recomendable en la actualidad. Teniendo en cuenta la importancia de las revistas científicas en la comunicación de los resultados de la investigación, se ha analizado el uso de este indicador en las publicaciones españolas editadas en acceso abierto, verificando como resultado una tendencia a propiciar el empleo del ORCID en estas

* Facultad de Comunicación y Documentación, Universidad de Murcia, España

javima@um.es rosanalc@um.es

INVESTIGACIÓN BIBLIOTECOLÓGICA, vol. 33, núm. 80, julio/septiembre, 2019, México, ISSN: 2448-8321 pp. 73-95 
publicaciones, debido a la facilidad de su adopción, a través de los sistemas de gestión editorial como OJS, su interoperabilidad y su presencia en las revistas de mayor impacto.

Palabras clave: Identificadores de Autor Digitales; Perfiles en Línea de Investigadores; Control de Autoridades; Identidad Digital; Índice h; Revistas Científicas; ORCID.

The gradual adoption of ORCID for improving the digital identity of scientific Spanish reviews in open access

Francisco Javier Martínez-Méndez and Rosana LópezCarreño

\section{Abstract}

This study reflects about the need to adopt a digital identifier for authors and / or researchers which facilitates the control of authorities, as well as updating the curriculums and determining the metrics of the scientific production. It concludes that ORCID may be the most recommended indicator today. Considering the importance of scientific journals in the communication of results, we have analyzed the use of this indicator in the open access Spanish journals and verified a tendency to encourage the use of ORCID in these publications due to the ease of its adoption through editorial content management systems such OJS, its interoperability and its presence within the most cited academic journals.

Key Words: Digital Author Identifiers; Researchers Online Profiles; Authority Control; Digital Identity; H-Index; Scientific Communication; Journals; ORCID.

\section{INTRODUCCIÓN}

1 control de autoridades es el proceso de unificar, mediante la utiliza-
ción de una forma normalizada, los puntos de acceso de los catálogos 
automatizados mostrando las relaciones entre los puntos de acceso (Herrero, 1999: 121). Hasta ahora, ha sido una necesidad constante en el ámbito bibliotecario para la normalización de la catalogación e indización, y como soporte de medición del impacto de los autores e investigadores, a partir de la aplicación de indicadores bibliométricos basados en el autor. En la actualidad sigue siendo un desafío la identificación correcta de los nombres de los autores. Es fundamental conseguir la desambiguación en la identificación de autores o investigadores, eliminando ruido y sesgos en las métricas vinculadas al impacto del autor (por ejemplo, los índices H o i10 de Google Scholar Citations). La identificación del autor o autores con alta precisión es vital para el proceso de datos bibliométricos (Krämer et al., 2017). Asociar debidamente los trabajos académicos con su autor o autores ha sido casi siempre un problema. Considerando otros avances obtenidos en la recuperación de información, surge la duda sobre si, finalmente, tenemos las herramientas necesarias para abordar este problema que presenta (entre otras), las siguientes facetas: formatos de nombres incoherentes causados por los propios autores o editores, presencia de varios sistemas de transliteración (especialmente con los nombres de alfabetos no latinos), posibles cambios legales de nombre (habitual en países anglosajones) y las distintas variantes culturales en la gestión de nombres compuestos y apellidos, incluyendo aquí la presencia de guiones (Wagner, 2009).

Desde el inicio de la difusión de la información científica por medio de las revistas, ha existido el problema de identificar claramente a los científicos que, con su esfuerzo, han contribuido al avance de sus disciplinas. Si bien las revistas científicas recogen los nombres de los autores contribuyentes, por lo general con afiliaciones institucionales, esta información a menudo es insuficiente para identificarlos de manera única, especialmente a lo largo del tiempo. La comunidad de investigadores científicos — de hecho, la comunidad académica como un todo- carece del equivalente funcional de un doi para gestionar la identidad académica, un identificador persistente que vincule de forma única a cualquier investigador con su corpus de contribuciones. Esto no significa que los sistemas de identidad académica no existan, quien haya publicado en una revista importante, depositado un trabajo en arXiv.org, solicitado financiación a una agencia promotora de la ciencia, o agregado contenido a un repositorio institucional suele poseer un identificador específico para esa plataforma. El problema es la "balcanización" de estas identidades, que hace casi imposible formar una visión completa de las contribuciones de una persona. Además, las compañías editoriales con fines de lucro que alojan las identidades de los académicos pueden intentar monetizar ese contenido, yendo en contra de la cultura abierta de la investigación científica (Evrard et al., 2015:2). 
Muchas plataformas y redes sociales académicas ofrecen métricas a nivel de autor. Según Orduna-Malea et al. (2017a), se dividen en cinco categorías: bibliométricas (publicación y citación), uso, participación, valoración y conectividad social. Todas las interacciones en la forma de visionados, descargas, lecturas, enlaces, comparticiones, menciones, revisiones, incrustado y etiquetado de contenidos, discusiones, marcadores, votos, seguimiento de usuarios, valoraciones y citas son rastreadas por la plataforma y transformadas en indicadores, a partir de los cuales los investigadores disponen de información sobre el impacto que sus trabajos tienen en las comunidades científicas y profesionales, así como en los medios de comunicación en general. Estos servicios permiten a los investigadores construir una presencia en línea cada vez más importante al evaluar las actividades de un investigador.

En un contexto de creciente competencia en la investigación académica, estas herramientas resultan ser formas efectivas para que los investigadores destaquen y aumenten sus posibilidades de progreso profesional y conseguir impacto científico (Greifeneder et al., 2018: 119). Por esta razón, plataformas como Researchgate, Publon y ORCID utilizan este argumento para alentar a los investigadores a participar: "los beneficios personales de tales prácticas continuarán siendo un importante motor en la transición hacia la ciencia abierta y pueden ser reforzados por el reconocimiento oficial de estas herramientas en el proceso de evaluación de los investigadores" (Crouzier, 2015:22).

Como indican Fernández-Marcial y González-Solar (2015), la identidad digital permite al investigador su identificación y reconocimiento en el contexto digital. Por esta razón, resulta muy interesante que su universidad o centro de investigación fomente, normalice y gestione esta identificación como parte de una estrategia institucional para mejorar su visibilidad global en el ámbito científico internacional, permitiendo a estas instituciones diferenciarse de otras universidades y percibir cómo se reconoce a sí misma, tanto internamente, como hacia afuera, en un contexto de cultura digital (Lara, 2009: 17). Dicha estrategia no sólo debe contemplar la normalización de perfiles para agilizar la propia gestión interna, sino que debe incluir otros objetivos también importantes, como la evaluación científica y la gestión editorial de las publicaciones propias. Smith-Yoshimura et al. (2014: 7) opinan que esta identificación unívoca de las autorías y su asociación correcta con los resultados de investigación ha hecho evolucionar a la bibliometría hacia la medida del impacto social de los trabajos, a partir de considerar las menciones en redes sociales y medios de comunicación en general (considerando no sólo las revistas científicas). Estos mismos autores recuerdan la trascendencia que han cobrado hoy en día los posicionamientos (rankings) de instituciones universitarias (en concreto citan el Times Higher Education, THE, 
World University Rankings, Academic Ranking of World Universities y QS Top Universities) en la imagen social de las universidades. Todas estas clasificaciones s consideran como fuente de datos las citas de los resultados de la investigación, y cuando una universidad no obtiene los resultados esperados en este aspecto, resulta norma común preguntarse si las principales bases de datos de citas han recopilado correctamente esa producción, a partir de una posible incorrecta identificación de los autores y de la afiliación institucional (muchos investigadores clínicos suelen indicar como lugar de trabajo un hospital o un laboratorio de investigación y obvian citar a la universidad a la que pertenecen).

Por tal razón, las universidades e instituciones de investigación deben intentar garantizar la identificación de los autores para la adecuada vinculación con la producción científica y, complementaria y necesariamente, verificar su correcta afiliación. En esto se basa el concepto de "reputación digital” que citan Fernández-Marcial y González-Solar (2015: 657) para quienes redunda en la sostenibilidad, competitividad y estabilidad de las universidades mediante su presencia en esos posicionamientos, asumiendo que para que una investigación sea visible, su calidad incorpora una nueva dimensión a partir de esa identidad. En el ecosistema digital en el que nos desenvolvemos, es totalmente necesario reforzar el control de autoridades, y es preciso apoyarlo debidamente en unos sistemas consistentes y permanentes de identificación y de perfiles de los investigadores, herramientas reconocidas para la visibilidad de la producción científica en todos los ámbitos que favorecen la medición del impacto, tanto individual como institucional.

Este refuerzo de la identidad digital se debe impulsar desde el seno de la gestión editorial de las publicaciones, con la adopción de nuevos formatos y políticas del proceso editorial, iniciado con la identificación unívoca de los autores/investigadores. Este trabajo tiene como objetivo identificar las herramientas más populares en la generación de perfiles e identidad digital y, si se puede hablar de una tendencia de uso, dentro de la gestión editorial de las revistas científicas en general, centrando el estudio de campo en el conjunto de revistas científicas españolas publicadas en acceso abierto.

\section{Sistemas de identificación y DE PERfILES DE AUTORES}

Los motores de búsqueda académicos (Google Académico o Microsoft Académico), las redes sociales académicas (ResearchGate, Academica.edu o Mendeley), las plataformas o bases de datos bibliográficas (Web of Science o Scopus) y los repositorios digitales científicos (Pubmed o ArXiv) han ajustado sus herramientas y servicios para ofrecer información actualizada sobre 
autores e investigadores, así como para la elaboración de perfiles avanzados y la agregación de información académica, ya sea a través de metadatos, ya sea importando registros por medio de otros identificadores externos. Fernández-Marcial y González-Solar (2015) distinguen entre:

- Plataformas de autoridades ("authority hubs") que proporcionan una ubicación centralizada para registros de autoridad de múltiples organizaciones (Researcher ID).

- Plataformas de identificadores ("ID hubs") que facilitan la creación de un registro centralizado de identificaciones (ORCID).

- Sistemas de gestión de referencias ("reference management systems") que ayudan a los investigadores organizar el trabajo, colaborar y descubrir nuevas publicaciones (Mendeley).

- Sistemas de perfiles de investigadores ("researcher profile systems") que posibilitan la creación de redes profesionales (Google Scholar Citations o ResearchGate).

Para Lorenzo-Escolar y Pastor-Ruiz (2012), el identificador de autor es un código (numérico o alfanumérico) que se asigna a un autor para identificar, de forma inequívoca, su producción científica, con independencia de cómo firma o en qué institución trabaja. El término "perfil de autor" se aplica al conjunto de datos que recogen de forma estandarizada, bien únicamente su producción científica o toda la actividad investigadora (puestos desempeñados, proyectos de investigación, contratos, etc.) de un autor. En un principio existían cuatro modalidades: Sistemas de Identificación Puros (SIP), Sistemas de Perfiles Puros (SPP), Sistemas Mixtos (SM), que aúnan identificador y perfil de autor $y$, finalmente, los Sistemas Globales, que aúnan un identificador y un perfil de autor de cualquier operador (SG).

\begin{tabular}{|l|l|l|}
\hline \multicolumn{1}{|c|}{ Tipo } & \multicolumn{1}{|c|}{ Nombre } & \multicolumn{1}{c|}{ Organización } \\
\hline SIP & $\begin{array}{l}\text { ISNI } \\
\text { IraLIS }\end{array}$ & $\begin{array}{l}\text { ISNI International } \\
\text { IraLIS. International }\end{array}$ \\
\hline SPP & LATTES & $\begin{array}{l}\text { National Council for Scientific and Technological Development } \\
\text { (CNPq) }\end{array}$ \\
\hline SM & $\begin{array}{l}\text { Researcher ID } \\
\text { Scopus Author ID }\end{array}$ & $\begin{array}{l}\text { Thomson Reuters } \\
\text { Elsevier }\end{array}$ \\
\hline
\end{tabular}




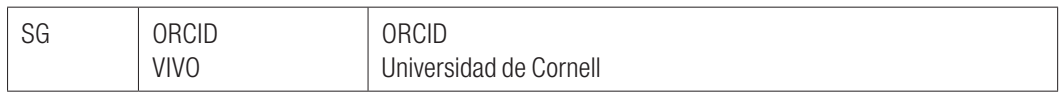

Cuadro 1. Clasificación inicial de los principales sistemas de identificación y perfil de autor. Fuente: Lorenzo-Escolar y Pastor-Ruiz (2012).

En los últimos años, este listado de sistemas de identificación y perfil de autor se ha visto ampliado sustancialmente por varios motivos:

- La gestión de las variantes de nombres y homónimos: nombres propios compuestos, apellidos compuestos, uso de determinantes, uso de la letra " $\tilde{n}$ " en el ámbito anglosajón, uso de partículas o determinantes, particularidades idiomáticas, etc. (Corchuelo-Rodríguez, 2014).

- El uso cada vez más frecuente de indicadores bibliométricos basados en el autor.

- El papel cada vez más importante de los identificadores únicos en la infraestructura editorial científica (Krämer et al., 2017).

- El aumento de la visibilidad e impacto de la producción científica de un investigador, con la apertura de un perfil de autor (Gasparyan et al., 2017).

- La cada vez mayor redifusión del trabajo científico en canales de comunicación no tradicionales, como preprints, blogs o redes sociales, ambientes que también precisan de métodos de identificación segura de la autoría (Crouzier, 2015: 23).

- Por la necesidad de establecer un Digital Author Identifer (DAI) único y universal, similar al DOI, que facilite la recuperación de información en cualquier plataforma o fuente de información (Gómez-Ontiveros, 2017).

El Cuadro 2 actualiza esta clasificación y distingue entre identificadores permanentes de autores (IDA), perfiles de autores/investigadores (PAS) y sistemas mixtos (SM) que combinan la identificación con el perfil del investigador, descartando la modalidad de sistema global (SG).

Existe una amplia gama de IDA y PAS que llegan a dificultar, sin duda alguna, la normalización si se da la circunstancia de no ser interoperables entre sí. Otro problema añadido es que el peso de la revisión y validación de la información que contienen recae exclusivamente sobre los propios autores o investigadores en la búsqueda de una mayor fiabilidad que necesita del pleno compromiso del autor (quien a veces no valora suficientemente este problema). Lo habitual es que el autor sea su propio gestor o administrador de su comunidad (community manager) científica", asumiendo así el papel de principal promotor y difusor de su producción científica. Esto no es del todo 
recomendable, ya que tiende a provocar la desactualización de los perfiles, debido a la escasez de tiempo para dedicarlo a esta tarea y a la diversidad de plataformas, redes y sistemas de identificación, en las cuales gestionar esa información, muchas de ellas emergentes, lo que conlleva una necesidad de aprendizaje suplementario que no siempre se realiza adecuadamente. Esto suele derivar en la introducción de sesgos y ruidos en la asignación de autorías de la producción científica, repercutiendo negativa y directamente en las métricas, así como en el impacto de su trabajo de investigación.

\begin{tabular}{|c|c|c|}
\hline Tiро & Nombre & Organización \\
\hline \multirow[t]{3}{*}{ IDA } & $\begin{array}{l}\text { ArXiv Author ID } \\
\text { Author Claim }\end{array}$ & $\begin{array}{l}\text { ArXiv } \\
\text { RePEc Author }\end{array}$ \\
\hline & $\begin{array}{l}\text { IraLIS } \\
\text { ISNI }\end{array}$ & $\begin{array}{l}\text { IraLIS. International } \\
\text { ISNI International }\end{array}$ \\
\hline & $\begin{array}{l}\text { VIAF } \\
\text { Academia.edu }\end{array}$ & $\begin{array}{l}\text { OCLC } \\
\text { Academia.edu }\end{array}$ \\
\hline \multirow{2}{*}{ PA } & $\begin{array}{l}\text { CVN } \\
\text { Google Scholar Citations }\end{array}$ & $\begin{array}{l}\text { FECyT } \\
\text { Google }\end{array}$ \\
\hline & $\begin{array}{l}\text { Kudos } \\
\text { LATTES }\end{array}$ & $\begin{array}{l}\text { Kudos } \\
\text { National Council for Scientific } \\
\text { and Technological Development } \\
\text { (CNPq) }\end{array}$ \\
\hline \multirow[t]{4}{*}{ SM } & $\begin{array}{l}\text { Microsoft Scholar Author } \\
\text { OpenScience }\end{array}$ & $\begin{array}{l}\text { Microsoft } \\
\text { OpenScience }\end{array}$ \\
\hline & $\begin{array}{l}\text { Publons } \\
\text { VIV0 }\end{array}$ & $\begin{array}{l}\text { Clarivate Analytics } \\
\text { Universidad de Cornell }\end{array}$ \\
\hline & $\begin{array}{l}\text { ORCID } \\
\text { Researcher ID }\end{array}$ & $\begin{array}{l}\text { ORCID } \\
\text { Thomson Reuters }\end{array}$ \\
\hline & $\begin{array}{l}\text { ResearchGate } \\
\text { Scopus Author ID }\end{array}$ & $\begin{array}{l}\text { ResearchGate } \\
\text { Elsevier }\end{array}$ \\
\hline
\end{tabular}

Cuadro 2. Principales sistemas de identificación y perfil de autor. Fuente: Elaboración propia (2018).

A pesar de que muchos de estos sistemas de identificación y perfil de investigadores se complementan entre sí, la infrautilización de algunos de ellos y los usos inadecuados (como es el caso de la promoción de revistas, mediante la creación de perfiles de revista en plataformas como ResearchGate, que está concebida para autores/investigadores), se encuentran entre sus limitaciones (Gasparyan et al., 2017). Son muchos los investigadores que ya tienen múltiples perfiles o ID, que pueden no estar vinculados. Un investigador puede llegar a tener perfiles o identificaciones 
en academia.edu, Google. Académico, ISNI, Mendeley, Microsoft Academic, ORCID, ResearchGate, Scopus, IAF y VIVO, por ejemplo, y, al mismo tiempo, tener una presencia fragmentada en la web. Algunos estudios consideran la necesidad de desarrollar mecanismos para asociar IDs (Smith-Yoshimura et al., 2014).

Algunos sistemas IDA, como IraLIS o ISNI, responden más a la necesidad del control de autoridades; mientras que algunos de los perfiles PAS, como Google Scholar Citations o Microsoft Scholar Author, están más enfocados a la trayectoria investigadora de un autor en aras de la divulgación científica personal. Se da la circunstancia de que estos perfiles ya citados han sido impulsados por los principales motores de búsqueda para fidelizar a los investigadores en el uso de sus herramientas de apoyo y a la autogestión de sus perfiles personales. Representan una opción harto interesante, pero carecen de filtros de control para que un autor no se asigne (por error o intencionadamente) trabajos que no son suyos, la presencia de elementos irrelevantes (no todo lo que se recopila se considera producción científica) y la inexistencia de un tesauro para sistematizar las búsquedas, limitan el buen funcionamiento de estos PAS provenientes de motores de búsqueda. No obstante, en el caso de Google Scholar, sus responsables trabajan en la introducción de herramientas de validación que mejoren la confiabilidad en sus perfiles. Otro dato que inevitablemente habla en favor de estos sistemas es el inmenso tamaño de la base de datos que los sustenta (en Google Scholar, el tamaño de su base de datos triplica a la de los índices de citas tradicionales).

La aparición de Publons (de Clarivate Analytics) amplía la función básica de identificación de autores hasta a la de identificación de expertos ("ID Review"), permitiendo así que los autores/investigadores puedan ser invitados a participar en comités editoriales para las tareas de revisión de los manuscritos. En este caso, los usuarios registrados cumplimentan sus perfiles con fotografías y notas biográficas, enumeran las revistas en las que ya participan como revisores $\mathrm{u}$ otras tareas editoriales e importan información desde ORCID u otros perfiles académicos. Los IDA surgen como herramientas normalizadoras de las variantes de nombres propios, de ahí que su utilidad, eminentemente enfocada a la recuperación de información, limita su empleo a prescripciones o políticas de publicación de los grandes grupos editoriales (Elsevier, Springer Nature, etc.), en los que se impone su uso a través de los sistemas de gestión editorial de sus revistas (por ejemplo ScholarOne de Clarivate Analytics). 
La fusión de los identificadores puros con los perfiles de autores/investigadores y revisores sería algo lógico y natural, además de deseable. Por ello, de entre la gama de fuentes de información de autoridades existentes se ha popularizado el uso de los sistemas mixtos de identificación y edición de perfiles de autores ORCID, RID (Research ID de Clarivate Analytics), RG (ResearchGate) y SAID (Scopus Author ID). De estos cuatros sistemas mixtos de identificadores, sólo la red social académica $(R G)$ no asigna un identificador unívoco, tal como hacen los otros tres sistemas, en su lugar emplea la URL del perfil del autor.

\begin{tabular}{|l|l|}
\hline $\begin{array}{l}\text { Sistema mixto } \\
\text { de identificación }\end{array}$ & \multicolumn{1}{|c|}{ Ejemplo } \\
\hline ORCID & $0000-0003-1098-9361$ \\
RID & C-8947-2011 \\
\hline RG & https://www.researchgate.net/profile/Francisco_Javier_Martinez-Mendez \\
SAID & 6506448438 \\
\hline
\end{tabular}

Cuadro 3. Ejemplo de codificación en sistemas mixtos de identificación y perfil de autor. Fuente: Elaboración propia (2018).

La adopción y posterior popularización entre la comunidad científica del indicador Índice b para medir el impacto científico de un autor/investigador, está desplazando paulatinamente el objeto de medida desde la revista (establecida desde los años sesenta y basada en el factor de impacto) hacia el autor/ investigador (la unidad de medida no cambia, sigue siendo la cita de un trabajo). Esto representa un punto de inflexión en la bibliometría y en la evaluación científica. En la Declaración sobre Evaluación de la Investigación de San Francisco (DORA), de 2013, se postulaba no apoyarse de manera incondicional en los indicadores de impacto a la hora de evaluar los logros de investigación.

Desde el punto de vista teórico, los factores de impacto de las revistas ya no serían tenidos tan en cuenta como hasta ahora, ni directa ni indirectamente en la evaluación del personal investigador (López et al., 2017). Esta propuesta es algo que reclama (por ahora sin mucho éxito) una buena parte de la comunidad científica internacional, tradicionalmente sometida a la evaluación de su actividad científica por la relevancia de la revista donde publica, no por la calidad de su trabajo.

El éxito de los sistemas mixtos de identificación y edición de perfiles de autoridades científicas no radica sólo en sus posibilidades de fusión o combinación (como es el caso de la integración de RID y ORCID, que permite 
actualizar el perfil de los autores a partir de sus datos, recogidos en la base de datos Web of Science, WoS), sino también en la incorporación de métricas basadas en el impacto de los autores entre otros indicadores "alternativos".

Nuestro interés se centra en el estudio de estos sistemas, de sus características y de sus usos. Las plataformas bibliográficas también integran herramientas de medición de impacto a nivel de artículo (el caso de Scopus con la integración de PlumX Metrics), tomando en consideración aspectos como el uso (descargas, visualizaciones), las capturas (marcadores), la mención de los trabajos científicos (publicaciones en blogs, comentarios electrónicos y referencias en Wikipedia), junto con la atención de las redes sociales, dando así un paso más allá de los meros recuentos de citas en revistas de cierto impacto (una de las reclamaciones clásicas de buena parte de la comunidad científica internacional).

Gasparyan et al. (2017) citan en su trabajo un análisis realizado sobre 6,132 perfiles de Google Scholar, Academia.edu, ResearchGate y Mendeley. Dicho estudio demostró que los investigadores de ciencias sociales y humanidades dependen principalmente de la red social científica Academia.edu, mientras que los biólogos prefieren usar ResearchGate como red social científica de referencia. Citan también una encuesta realizada a 296 miembros de una de las facultades más prestigiosas de Nueva York, que mostraba como resultado una sorprendentemente baja conciencia del uso de los identificadores de autor (sólo un 32 por ciento), siendo su uso un poco mayor en las áreas de física, biología, ciencias de la salud y matemáticas que en el resto de disciplinas, Los investigadores encuestados usaban principalmente el ID de ORCID ( $n=49,15$ por ciento), seguidos de la ID de autor de Scopus ( $n=29,9$ por ciento), ID de investigador ( $n=25,7$ por ciento) e ID de $\operatorname{ArXiv}(n=20,6$ por ciento).

Como ya se indicó, los sistemas mixtos más populares son los comerciales RID y SAID, junto con los abiertos ORCID y ResearchGate. Casi todos precisan de la autoedición por parte de los usuarios de sus perfiles correspondientes, salvo en el caso de SAID, que la lleva a cabo automáticamente, a partir de los datos que posee esta base de datos de los autores (RID también integra datos de otras fuentes de la plataforma WoS, pero sigue precisando de la gestión manual del perfil). Entre los sistemas que posibilitan la gestión y edición del propio perfil del autor, algunos permiten también indicar las variedades de nombres por las que un autor puede ser indizado, posibilitando así un más extenso control de autoridades, sin pérdida de precisión en la autentificación del autor y un recuento de citas más eficaz y completo.

Respecto de la interoperabilidad de los sistemas de identificación y perfilado de la currícula de investigadores con el contenido de las distintas bases de datos bibliográficas y de citas, más específicamente en todo lo relacionado 
con la posibilidad de extraer datos de manera automática, dichos sistemas permiten mayor agilidad en las tareas de autogestión y cumplimentación de los perfiles investigadores. En este punto en concreto, ORCID es el sistema que mayores prestaciones posee, ya que está "integrado en grandes plataformas y bases de datos bibliográficas, así como en sistemas de gestión editorial de revistas como OJS (Open Journal Systems) o ScholarOne" (Gasparyan et al., 2017), poseyendo más posibilidades de interoperabilidad que otras plataformas similares, como ISNI o ResearcherID (Lee, 2016).

\begin{tabular}{|l|c|c|c|l|l|}
\hline $\begin{array}{c}\text { Sistema } \\
\text { mixto }\end{array}$ & $\begin{array}{c}\text { No. de } \\
\text { usuarios reg. }\end{array}$ & Autoedición & $\begin{array}{c}\text { Control } \\
\text { de autoridades }\end{array}$ & Interoperabilidad & $\begin{array}{l}\text { Compatibilidad } \\
\text { con otros IDS/PAS }\end{array}$ \\
\hline ORCID & $4,500,000$ & $X$ & & $\begin{array}{l}\text { Crossref } \\
\text { DataCite } \\
\text { Emerald Group } \\
\text { Publishing } \\
\text { Impact story } \\
\text { Pubmed } \\
\text { Redalyc } \\
\text { Scopus }\end{array}$ & $\begin{array}{l}\text { Academia.edu } \\
\text { Kudos } \\
\text { Publons } \\
\text { ResearcherlD } \\
\text { ResearchGate } \\
\text { Scopus Author } \\
\text { ID }\end{array}$ \\
\hline RID & 108,000 & $X$ & $X$ & $\begin{array}{l}\text { Publons } \\
\text { Web of Science }\end{array}$ & ORCID \\
\hline RG & $14,000,000$ & $X$ & $X$ & $\begin{array}{l}\text { ArXiv } \\
\text { CiteSeer } \\
\text { PubMed }\end{array}$ & ORCID \\
\hline SAID & $12,000,000$ & & & Scopus & CVN \\
\hline
\end{tabular}

Cuadro 4. Características y usos de sistemas de identificación y perfil de autor.

Fuente: Elaboración propia (2018).

El Cuadro 5 resume el Cuadro 4, presentando los sistemas que más destacan por parámetro de medida analizado:

\begin{tabular}{|l|c|}
\hline \multicolumn{1}{|c|}{ No. de usuarios registrados } & $R G$ \\
\hline Autoedición & ORCID, RID y RG \\
\hline Control de autoridades & RID y SAID \\
\hline Interoperabilidad & ORCID \\
\hline Compatibilidad & ORCID \\
\hline
\end{tabular}

Cuadro 5. Sistema más utilizado en función del parámetro analizado.

Fuente: Elaboración propia (2018). 
Si bien RG es el sistema con mayor difusión en estos momentos (el volumen de la red social académica que lo sustenta ayuda mucho a posicionarlo en el primer lugar), la interoperabilidad y la compatibilidad colocan a ORCID en posición de ventaja, siguiendo los consejos de los autores que apuestan por una mayor interconexión entre los distintos sistemas. En cuanto al control de autoridades, ambos sistemas deben mejorar sus prestaciones. En un nivel inferior aparece el sistema RID, que además se emplea en una base de datos comercial y tiene, por tanto, menor alcance en el acceso abierto. Un punto fuerte de los sistemas mixtos de identificación y perfil de autores es la compatibilidad con otros identificadores y perfiles externos. Así se facilita el filtrado de los datos de un investigador, permitiendo a las plataformas bibliográficas (de las cuales dependen algunos de estos identificadores), expandir abiertamente la divulgación científica de los autores, sin incidir en el modelo de negocio de la plataforma ni de la revista científica, generando, a su vez, una amplia red de identificadores de investigadores. También, en este aspecto, ORCID se presenta como el sistema más adecuado.

La integración de ORCID como identificador permanente obligatorio para los autores en el registro de sus manuscritos es algo cada vez más habitual. No sólo facilita el control de autoridades a las plataformas bibliográficas que dan soporte a las revistas, también permite transferir registros bibliográficos desde estas plataformas hacia el propio perfil ORCID del autor/investigador, tal como ocurre en Pubmed, CrossRef o Scopus. De esta forma, se lleva a cabo una transacción multidireccional de datos revisados por los propios autores. La clave del éxito de ORCID residiría quizás en la inserción y uso obligatorio de este identificador en OJS (el sistema de gestión editorial de revistas científicas diseñado y distribuido por Public Knowledge Project), el sistema de gestión de contenidos para la edición de revistas científicas "open source" más implantado (Delgado-Vázquez, 2018). El número de instalaciones superaba los nueve mil en 2017, la mayor parte de las cuales apuestan por el acceso abierto a la información. En su última versión (OJS 3.1.1-2), este gestor de contenidos permite su uso en el proceso editorial de una revista y, además, puede configurarse como elemento obligatorio para el registro de autores y revisores, opción sumamente interesante para los editores de estas revistas, ya que favorece la interoperabilidad de sus contenidos científicos, aumentando su difusión e impacto. 
Es interesante que los sistemas de identificación permanentes y de gestión de perfiles de investigadores establezcan sus propios indicadores, combinando indicadores básicos de citas (como el Índice h) con otros sobre uso y actividad del propio perfil (aunque en ocasiones sean sospechosos de falta de transparencia en sus aplicaciones y se descarten como medidores para evaluación científica). Este índice (Hirsch, 2005) se ha convertido en uno de los indicadores bibliométricos más empleados para calcular el éxito del trabajo realizado por un investigador y predecir el impacto de su producción en el futuro. Esta tendencia se debe, principalmente, a dos razones: en primer lugar, a su simplicidad (se trata de un único indicador que combina producción e impacto y que resulta fácil de explicar) y porque puede ser determinado fácilmente por cualquier investigador; en segundo lugar, tiene la ventaja de eliminar los sesgos provocados por las colas de la distribución de citas (Dorta-González y Dorta-González, 2010). No obstante, presenta algunas limitaciones: beneficia a los grandes productores frente a aquellos más selectivos (ibíd., 2010); no sirve para comparar autores de disciplinas científicas diferentes y también existen problemas técnicos para obtener todos los artículos de un autor a causa de la similitud de nombres y apellidos. Por ello, se está trabajando en su uso complementario con otro indicador de "entorno", que contextualice la producción científica de un autor dentro de su área de trabajo "al incluir un volumen de citas adicional mayor en el caso de los autores selectivos" (Dorta-González y Dorta-González, 2010). De todos modos, es innegable que se ha convertido en un importante competidor del tradicional factor de impacto de las revistas científicas. En su popularización ha tenido mucho que ver su empleo en la base de datos Web of Science (a través de RID) y en la base de datos Scopus, si bien lo decisivo es su presencia, mucho más abierta y accesible para toda la comunidad científica, en el buscador académico Google Scholar (vía Google Scholar Citations).

Como indican Orduna-Malea et al. (2017b), Google Scholar y su producto derivado Google Scholar Citations presentan algunos problemas de fiabilidad en la agregación automática de registros bibliográficos asignados a cada investigador, lo que provoca cierto rechazo y crítica por una parte de la comunidad científica. Esto debido, fundamentalmente, a la falta de normalización e identificación de los autores, supeditada a la indización de origen de las fuentes de información de las que Google Scholar extrae dichos registros, lo cual incide directamente en sus indicadores Índice b e Índice i10, y puede llegar a desvirtuar los promedios y clasificaciones de impacto 
de los investigadores (obligándolos a más revisiones de las deseadas de su perfil, si quieren tener una completa seguridad en sus datos). Esta cuestión no es trivial, porque existen clasificaciones que miden el impacto y difusión de las universidades en esta plataforma y que penalizan a los perfiles de autor que "acogen" citas a trabajos que no son de su autoría (es el caso del índice Transparent Ranking, elaborado por webometric.info, y que ha excluido en la edición de 2018 a varias universidades persistentes en este error).

Todo lo anterior evidencia la necesidad del control de autoridades para un exacto conteo de citas y, por tanto, un correcto uso métrico de la valoración de la producción científica. Una de las posibles soluciones sería la adopción de un identificador permanente, que factiblemente sería ORCID, para permitir a Google Scholar mejorar el filtrado y la asignación de la producción científica de cada autor.

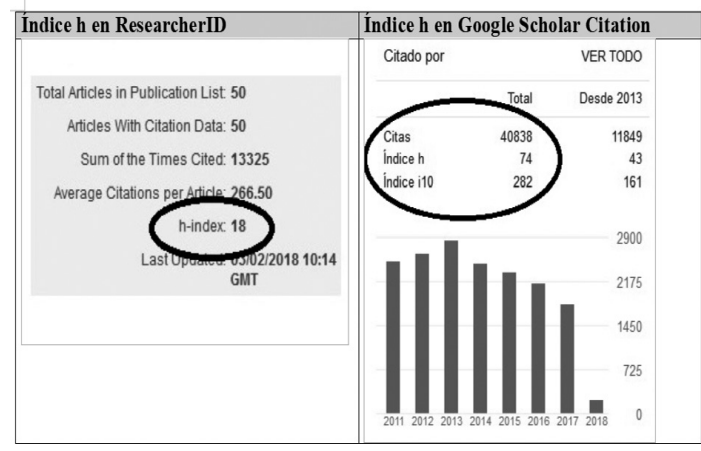

Figura 1. Ejemplos de usos de Índice h. Fuente: Elaboración propia (2018).

\section{APUESTA POR ORCID COMO SISTEMA GESTOR DE LA IDENTIDAD} DIGITAL EN LAS REVISTAS CIENTÍFICAS

La investigación científica es cada vez más multidisciplinar y colaborativa en el plano internacional, así, el descubrimiento de la atribución de logros individuales cada vez es más difícil. Parece claro que se requiere una solución a la identidad académica única y persistente, y el identificador ORCID es una solución disponible, abierta, no comercial, interdisciplinar y de alcance global. Varias sociedades y comunidades científicas en los campos de física y la astronomía lo respaldan activamente. Los beneficios a la comunidad científica son muy valiosos para ignorarlos (Evrard et al., 2015: 11). Otro aspecto favorable 
de ORCID es su estabilidad, lo cual permite controlar las autoridades en las revistas científicas (edición y publicación) y en todos los registros de autoridad (bases de datos bibliográficas y de patentes), a lo largo de toda su carrera académica. Su uso está cada vez más estandarizado. Ansley (2014: 679) considera que ORCID contribuye a resolver el problema de ambigüedad del nombre en la investigación y las comunicaciones académicas, por medio de un registro central de identificadores únicos para investigadores individuales, junto con un mecanismo de enlace abierto y transparente entre este sistema y otros esquemas de identificación de investigadores. Estos identificadores, y las relaciones entre sí, pueden vincularse con los resultados del investigador para mejorar el proceso de descubrimiento científico, contribuyendo paulatinamente a mejorar el control de autoridades (uno de los aspectos en los que vimos anteriormente que aún no destaca ORCID).

Harseim y Goodey (editor y analista de Springer Nature, respectivamente), encuestaron a casi tres mil investigadores de humanidades y ciencias sociales sobre su presencia digital en redes sociales y un 75 por ciento de ellos afirmaron usar este identificador (Staniland, 2017). Allen, (directora de evaluación de Wellcome Trust, organización de investigación biomédica británica), considera que ORCID se está convirtiendo rápidamente en el sistema predeterminado y piensa que los científicos deben registrarse para obtener una identificación al principio de su carrera y esforzarse por mantener sus perfiles actualizados (Schiermeier, 2015). De esta forma, el sistema ayuda al investigador novel a ubicarse dentro de su ecosistema científico de una forma rápida y eficaz, permitiendo distinguir su tendencia investigadora. Masic y Begic (2016) piensan que el uso de este identificador se impondrá obligatoriamente por parte de las revistas científicas (cuando escribían su artículo era ya utilizado por la British Library, y líderes editoriales como Elsevier, Springer Nature y Group and Dove Press) debido, fundamentalmente, a que aporta soluciones a las defectuosas transcripciones de nombres complejos, omisión de nombres intermedios y de iniciales, cambios en el nombre producidos por matrimonios o divorcios, así como la coincidencia de nombres comunes, uno de los principales problemas de Google Scholar Profiles y que ORCID contribuye a paliar.

A partir de estos planteamientos, hemos considerado interesante analizar el uso de este indicador por parte de las revistas españolas en abierto (si bien su uso no es exclusivo de este tipo de publicaciones, ya se indicó antes que, si bien las principales editoriales científicas comerciales tienen sus propios sistemas de identificación, existe una cierta integración con este identificador), con la idea de verificar si se han confirmado los buenos pronósticos que se habían hecho dentro del contexto editorial. Tomando como fuente 
de referencia el informe srj 2016 (Scimago Journal and Country Rank) por tener mayor alcance en indización de revistas abiertas que satisfacen severos criterios de calidad, se procedió a elaborar un listado de las 211 revistas científicas españolas editadas en acceso abierto, accediendo a todas (marzo de 2018) y verificando en qué grado se había incorporado ORCID para el control de sus autores.

El resultado obtenido no fue, a primera vista, muy alentador. Si bien las revistas científicas analizadas comienzan a apostar por el uso de identificadores digitales o permanentes, solo un 20.37 por ciento del total utilizan ORCID para estos fines. Si analizamos la distribución de las revistas por cuartil dentro del informe SJR, se observa que más de la mitad de estas revistas están situadas en el primer y segundo cuartil de sus respectivas categorías y, más concretamente, las revistas que emplean ORCID son mayoría en el primero de los cuartiles (6 de 11). Creemos que el hecho de que las revistas que mejor respetan las buenas prácticas editoriales y reciben más citas por parte de la comunidad científica usen este identificador, contribuirá positivamente a su implantación en breve plazo en una cantidad mayor de revistas, pudiendo llegar a considerarse en un criterio de calidad o de buenas prácticas en la edición de estas publicaciones científicas.

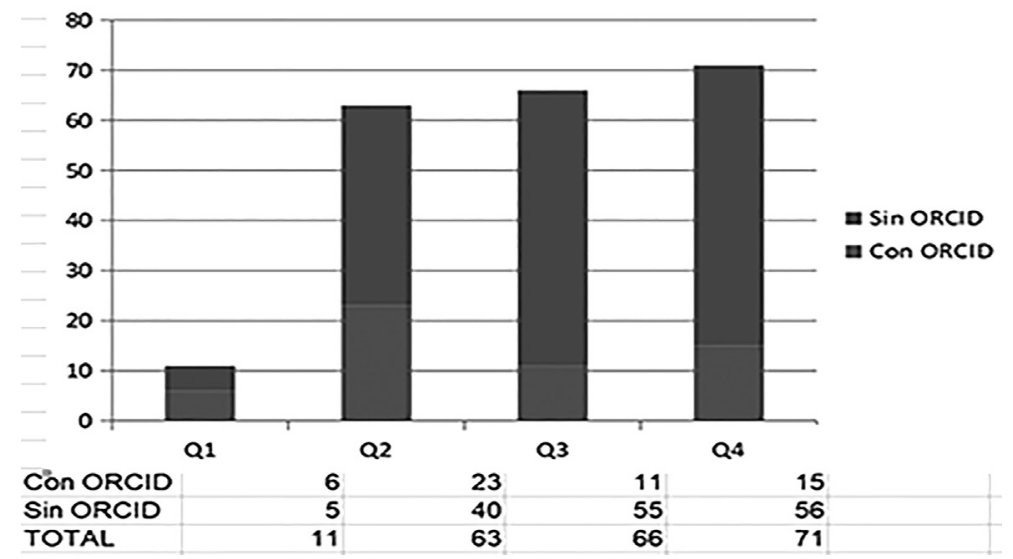

Figura 2. Posicionamiento de las revistas españolas de acceso abierto según SJR 2016.

Fuente: Elaboración propia.

También ayudará el hecho de que la mayoría de los editores hagan uso del gestor de contenidos Open Journal System (OJS), el cual permite una sencilla aplicación de ORCID y que apuesta por su uso, si bien ahora no disponemos de datos concretos, porque muchas de estas revistas no han ejecutado aún la 
última versión de este gestor de contenidos. Estamos convencidos de que la nueva versión de este sistema (presentada en septiembre de 2016 y adaptada a la nueva normativa de protección de datos en febrero de 2018) implicará un aumento de este porcentaje, a medida que los editores se actualicen con la nueva versión. En la Figura 3 se observa claramente que el año 2017 es el que marca el inicio del uso de ORCID entre las revistas científicas españolas analizadas (se han conseguido datos de 39 de las 43 publicaciones aquí estudiadas).

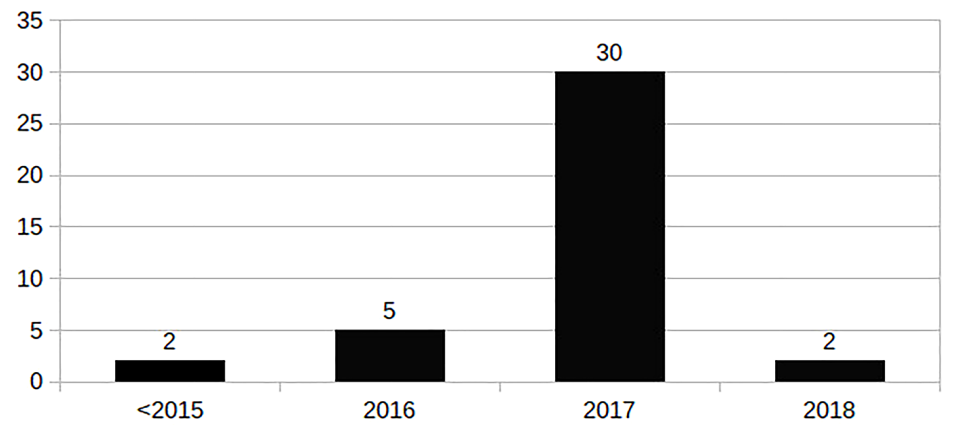

Figura 3. Año de inicio del uso de ORCID en las revistas españolas científicas de acceso abierto. Fuente: Elaboración propia.

Del mismo modo que ocurrió a partir del año 2012 con la adopción del sistema DOI (Digital Object Identifier), para identificar de manera unívoca los artículos publicados en revistas científicas, estamos convencidos de que se adoptará un identificador digital de autor, que creemos puede ser ORCID, a partir de los datos que hemos observado, debido a las prestaciones e integraciones en distintos sistemas o plataformas bibliográficas y su compatibilidad con otros identificadores similares (varios de los autores anteriormente citados, como Masic y Begic, Smith-Yoshimura et al. o Staniland así lo afirman).

Las políticas editoriales de los servicios de publicaciones de universidades y centros de investigación, sobre todo de carácter público, tienen la responsabilidad de adoptar un identificador digital para sus autores, no sólo para el control bibliográfico y uso bibliométrico, sino para garantizar la visibilidad internacional de sus investigadores y de sus instituciones. En el caso particular de las revistas científicas españolas que usan ORCID, 35 de las 43 son editadas por la principal institución científica de España: el Consejo Superior de Investigaciones Científicas. Si el resto de instituciones editoras toman este hecho como referencia, estamos seguros del aumento del uso de este identificador entre el conjunto de las revistas españolas de acceso abierto a la información, convirtiéndose en el indicador de referencia. 


\section{Conclusiones}

La falta de normalización de los nombres propios de los autores supone un hándicap en la medición bibliográfica, El Índice b está supeditado al control de autoridades, el cual se solventará a través del establecimiento de un identificador de autor digital único y universal, el cual permita interactuar con cualquier otro y que sea prescrito desde los sistemas gestión editorial. ORCID puede constituirse en ese identificador digital de autor único y universal, debido fundamentalmente a su integración e interoperabilidad en plataformas bibliográficas, gestores editoriales y otros perfiles de autores e investigadores. Parte de su éxito dependerá de su integración en Google Académico y sus derivados, además de sus buenas prestaciones en cuanto a la interoperabilidad.

Se evidencia una necesaria revisión de los indicadores basados en el autor para validar su constitución como unidad de medida del impacto científico de un investigador, lo que supondrá un avance en la equidad y ponderación de la evaluación científica. La fiabilidad y veracidad de los perfiles no puede basarse en la autogestión de los autores, la falta de actualización los debilitaría como fuente de información personal. Debe asumirse como una tarea de gestión institucional.

La incorporación de identificadores digitales de autores en las revistas científicas permitirá un control de autoridades exhaustivo, que a su vez repercutirá positivamente en el conteo y medición de la producción científica de los autores, lo que supondrá una mejora en equidad. Para muchos autores, ORCID se postula como el identificador digital de autor por el que opten las revistas científicas para gestionar y normalizar los nombres propios de sus autores, dotando a la publicación de un nuevo criterio de calidad, del mismo modo que ocurre con el uso del DOI.

El estudio de campo realizado en las revistas españolas de acceso abierto muestra (con datos de 2016) una pequeña porción de publicaciones que lo han incorporado. No obstante, el hecho de que el uso de ORCID se haya extendido en las revistas que ocupan los principales cuartiles y de que el principal editor científico de España (CSIC) lo haya adoptado como normal, permiten aventurar una paulatina incorporación de este identificador en la gestión editorial de estas revistas, pauta a seguir por otros tantos editores científicos. 


\section{REFERENCIAS}

Ansley, J. 2014. "How can we be certain who authors really are? Why ORCID is important to the British Journal of Dermatology". British Journal of Dermatology, 171: 679-680. DOI: 10.1111/bjd.13381.

Corchuelo-Rodríguez, C.A. 2014. "Bibliometría: análisis del índice H, los identificadores persistentes de autor y su aplicación en la comunidad científica colombiana”. Bogotá: Universidad de la Salle, tesis de Doctorado en Sistemas de Información, Bibliotecología y Archivística, en <http://eprints.rclis.org/24678/>.

Crouzier, T. 2015. Science Ecosystem 2.0: How will change occur? Bruselas: European Commision, en <https://ec.europa.eu/research/innovation-union/pdf/expert-groups/rise/ science_ecosystem_2.0-how_will_change_occur_crouzier_072015.pdf > .

Delgado-Vázquez. A.M. 2018. Gestión y edición de revistas académicas con software libre. El uso de Open Journal Systems 3. Madrid: Editum, en <http://libros.um.es/ editum/catalog/book/2061>.

Dorta-González, P. y M.I. Dorta-González. 2010. "Indicador bibliométrico basado en el índice h". Revista Española de Documentación Científica, 33 (2): 225-245, en $<$ https://doi.org/10.3989/redc.2010.2.733>.

Evrard, A. E., C. Erdmann, J. Holmquist, J. Damon y D. Dietrich. 2015. “Persistent, global identity for scientists". ArXiv: 1502.06274.

Fernández-Marcial, V. y L. González-Solar. 2017. "Servicios a la investigación en la biblioteca universitaria: gestión de la identidad digital”. Comunicação e Transformações Sociais, IX Congreso da Sopcom (12-14 de novembro de 2015). Coimbra: Associação Portuguesa de Ciências da Comunicação, vol. 1: 10-23.

Fernández-Marcial, V. y L. González-Solar, L. 2015. "Promoción de la investigación e identidad digital: el caso de la Universidade da Coruña". El profesional de la información, 24 (5): 656-664, en <http://www.elprofesionaldelainformacion.com/ contenidos/2015/sep/14.pdf $>$.

Gasparyan, A.Y., B. Nurmashev, M. Yessirkepov, D.A. Endovitskiy, A.A. Voronov y G.D. Kitas. 2017. "Researcher and author profiles: opportunities, advantages, and limitations". Journal of Korean Medical Science, 32 (11): 1749-1756, en <https://www.ncbi.nlm.nih.gov/pmc/articles/PMC5639053/>.

Greifeneder, E.; S. Pontis, A. Blandford, H. Attalla, D. Neal y K. Schlebbe. 2018. "Researchers' attitudes towards the use of social networking sites". Journal of Documentation, 74 (1): 119-136, en <https://doi.org/10.1108/JD-04-2017-0051>.

Gómez-Ontiveros, L.V. 2017. “Open Researcher and Contributor ID (ORCID)”. Revista Biomédica, 28 (1), en <http://revistabiomedica.mx/index.php/revbiomed/ article/view/551>.

Herrero Pascual, C. 1999. "El control de autoridades". Anales de Documentación, no. 2: 121-136, en <http://revistas.um.es/analesdoc/article/view/2621/2601>.

Hirsch, J.E. 2005. "An index to quantify an individual's scientific research output". ArXiv: physics/0508025v5.

Krämer, T., F. Momeni y P. Mayr. 2017. "Coverage of Author Identifiers in Web of Science and Scopus”. ArXiv: 1703.01319.

Lara, T. 2009. "El papel de la universidad en la construcción de su identidad digital". Revista de Universidad y Sociedad del Conocimiento 6 (1): 15-21. 
Lee, D.J. 2016. Authority Control for tamu Digital Asset Management Ecosystems, en $<$ http://hdl.handle.net/1969.1/157180>.

López, A. F., J. Sanz-Valero y J.M.C. Fernández. 2017. "El factor de impacto ya no es el patrón oro; la declaración de San Francisco sobre evaluación de la investigación". Journal of Negative and No Positive Results (JONNPR) 2 (5): 173-176, en <http://revistas.proeditio.com/jonnpr/article/view/1392>.

Lorenzo-Escolar, N. y F. Pastor-Ruiz. 2012. "Un análisis de los principales sistemas de identificación y perfil para el personal investigador". Aula Abierta 40 (2): 97108, en <https://dialnet.unirioja.es/servlet/articulo? codigo=3921021>.

Masic, I. y E. Begic. 2016. "Evaluation of scientific journal validity, it's articles and their authors. Unifying the Applications and Foundations of Biomedical and Health Informatics": 9-14. DOI: 10.3233/978-1-61499-664-4-9.

Orduna-Malea, E., A. Martín-Martín y E. Delgado López-Cózar. 2017a. "Métricas en perfiles académicos: ¿un nuevo juego adictivo para los investigadores?” Revista Española de Salud Pública 90, e20006, en <http://scielo.isciii.es/pdf/resp/ v90/1135-5727-resp-90-e20006.pdf>.

Orduna-Malea, E., A. Martín-Martín y E. Delgado López-Cózar. 2017b. “Google Scholar como una fuente de evaluación científica: una revisión bibliográfica sobre errores de la base de datos". Revista Española de Documentación Cientifica, 40 (4), en <https://doi.org/10.3989/redc.2017.4.1500>.

Schiermeier, Q. 2015. “Research profiles: A tag of one's own”. Nature, no. 526: 281-283, en <https:/www.nature.com/naturejobs/science/articles/10.1038/nj7572-281a>.

Smith-Yoshimura, K., M. Altman, M. Conlon, A.L. Cristán, L. Dawson, J. Dunham y P. Schreur. 2014. "Registering researchers in authority files". OCLC Research, en <https://pub.uni-bielefeld.de/download/2706030/2706035>.

Staniland, M. 2017. "How do researchers use social media and scholarly collaboration networks (SCNs)?" Of schemes and memes: A community blog from nature. com, en <http://blogs.nature.com/ofschemesandmemes/2017/06/15/how-do-researchers-use-social-media-and-scholarly-collaboration-networks-scns $>$.

Wagner, A. B. 2009. "Author Identification Systems [Tips from the Experts]", en <https://ubir.buffalo.edu/xmlui/bitstream/handle/10477/12772/Author\%20 Identification $\% 20$ Systems.pdf? sequence $=1>$. 
Revistas españolas en acceso abierto que utilizan ORCID

\begin{tabular}{|c|c|c|c|c|}
\hline $\begin{array}{l}\text { No. de } \\
\text { categoría }\end{array}$ & Título & Año ORCID & $S J R$ & Cuartil \\
\hline 1 & Comunicar & 2009 & 1. 162 & Q1 \\
\hline 3 & Trabajos de Prehistoria & 2017 & 0.902 & Q1 \\
\hline 4 & Revista Latina de Comunicación Social & 2013 & 0.823 & Q1 \\
\hline 47 & Informes de la Construcción & 2017 & 0.301 & Q1 \\
\hline 59 & Gladius & 2016 & 0.241 & Q1 \\
\hline 60 & América Latina Hoy & $x$ & 0.239 & Q1 \\
\hline 11 & Scientia Marina & 2017 & 0.537 & Q2 \\
\hline 13 & Materiales de Construcción & 2017 & 0.535 & Q2 \\
\hline 25 & Grasas y Aceites & 2017 & 0.404 & Q2 \\
\hline 26 & Pharmacy Practice & 2016 & 0.404 & Q2 \\
\hline 27 & Collectanea Botanica & 2017 & 0.395 & Q2 \\
\hline 29 & Documents d'Analisi Geografica & 2018 & 0.385 & Q2 \\
\hline 33 & Revista Complutense de Educación & $x$ & 0.361 & Q2 \\
\hline 36 & Journal of Applied Biomedicine & $x$ & 0.35 & Q2 \\
\hline 38 & Revista Española de Documentación Científica & 2017 & 0.347 & Q2 \\
\hline 42 & $\begin{array}{l}\text { Journal of Industrial Engineering } \\
\text { and Management }\end{array}$ & 2016 & 0.315 & Q2 \\
\hline 85 & Anuario de Estudios Americanos & 2016 & 0.194 & Q2 \\
\hline 103 & Anuario de Estudios Medievales & 2017 & 0.169 & Q2 \\
\hline 107 & Isegoria & 2017 & 0.16 & Q2 \\
\hline 116 & Revista de Indias & 2017 & 0.15 & Q2 \\
\hline 117 & Archivo Español de Arqueología & 2017 & 0.148 & Q2 \\
\hline 118 & Hispania Sacra & 2017 & 0.145 & Q2 \\
\hline 124 & Arbor & 2017 & 0.138 & Q2 \\
\hline 139 & $\begin{array}{l}\text { Revista de Dialectología y Tradiciones } \\
\text { Populares }\end{array}$ & 2017 & 0.127 & Q2 \\
\hline 145 & Sefarad & 2017 & 0.123 & Q2 \\
\hline 146 & Archivo Español de Arte & 2017 & 0.123 & Q2 \\
\hline 158 & Artnodes & $x$ & 0.113 & Q2 \\
\hline 175 & Al-Qantara & 2017 & 0.107 & Q2 \\
\hline 17 & Journal of Optometry & $x$ & 0.511 & Q3 \\
\hline 28 & Revista Española de Enfermedades Digestivas & X & 0.391 & Q3 \\
\hline
\end{tabular}




\begin{tabular}{|c|c|c|c|c|}
\hline 48 & Estudios Geológicos & 2017 & 0.299 & Q3 \\
\hline 54 & Anales del Jardín Botánico de Madrid & 2017 & 0.259 & Q3 \\
\hline 66 & Graellsia & 2017 & 0.233 & Q3 \\
\hline 81 & Architecture, City and Environment & $x$ & 0.198 & Q3 \\
\hline 89 & $\begin{array}{l}\text { Boletín de la Asociación Internacional de } \\
\text { Derecho Cooperativo }\end{array}$ & $x$ & 0.187 & Q3 \\
\hline 97 & Revista Internacional de Sociología & 2017 & 0.174 & Q3 \\
\hline 127 & Asclepio & 2017 & 0.136 & Q3 \\
\hline 174 & Catalan Journal of Linguistics & 2017 & 0.107 & Q3 \\
\hline 93 & Pirineos & 2017 & 0.184 & Q4 \\
\hline 104 & Revista de Metalurgia & 2017 & 0.164 & Q4 \\
\hline 129 & Prisma Social & 2017 & 0.134 & Q4 \\
\hline 137 & Estudios Geográficos & 2017 & 0.128 & Q4 \\
\hline 142 & $\begin{array}{l}\text { Revista General de Información } \\
\text { y Documentación }\end{array}$ & $x$ & 0.126 & Q4 \\
\hline 161 & Journal of Technology and Science Education & 2016 & 0.113 & Q4 \\
\hline 169 & Anales de Documentación & 2018 & 0.111 & Q4 \\
\hline 176 & Papers: Revista de Sociología & $x$ & 0.105 & Q4 \\
\hline 181 & Revista de Lingüística y Lenguas Aplicadas & $x$ & 0.102 & Q4 \\
\hline 188 & Philosophical Readings & $x$ & 0.101 & Q4 \\
\hline 192 & Cuadernos de Estudios Gallegos & 2017 & 0.101 & Q4 \\
\hline 195 & Arqueología de la Arquitectura & 2017 & 0.101 & Q4 \\
\hline 197 & Anuario Musical & 2017 & 0.101 & Q4 \\
\hline 204 & Revista de Literatura & 2017 & 0.1 & Q4 \\
\hline
\end{tabular}

Para citar este texto:

Martínez-Méndez, Francisco Javier y Rosana López-Carreño. 2019. "La paulatina adopción de ORCID para la mejora de la identidad digital de las revistas científicas españolas en acceso abierto". Investigación Bibliotecológica: archivonomía, bibliotecología e información 33 (80): 73-95. http://dx.doi.org/10.22201/iibi.24488321xe.2019.80.57994 
